\title{
Supersymmetry and the Anomalous Anomalous Magnetic Moment of the Muon
}

\author{
Jonathan L. Feng ${ }^{1}$ and Konstantin T. Matchev ${ }^{2}$ \\ ${ }^{1}$ Center for Theoretical Physics, Massachusetts Institute of Technology, Cambridge, Massachusetts 02139 \\ ${ }^{2}$ Theory Division, CERN, CH-1211, Geneva 23, Switzerland
}

(Received 14 February 2001)

\begin{abstract}
The recently reported measurement of the muon's anomalous magnetic moment differs from the standard model prediction by $2.6 \sigma$. We examine the implications of this discrepancy for supersymmetry. Deviations of the reported magnitude are generic in supersymmetric theories. Based on the new result, we derive model-independent upper bounds on the masses of observable supersymmetric particles. We also examine several model frameworks. The sign of the reported deviation is as predicted in many simple models, but disfavors anomaly-mediated supersymmetry breaking.
\end{abstract}

\section{DOI: $10.1103 /$ PhysRevLett.86.3480}

Measurements of spin magnetic dipole moments have a rich history as harbingers of profound progress in particle physics. In the leptonic sector, the electron's gyromagnetic ratio $g_{e} \approx 2$ pointed the way toward Dirac's theory of the electron. Later, the electron's anomalous magnetic moment $a_{e} \equiv\left(g_{e}-2\right) / 2 \approx \alpha / 2 \pi$ played an important role in the development of quantum electrodynamics and renormalization. Since then, increasingly precise measurements have become sensitive both to very high order effects in quantum electrodynamics and to hadronic processes, and the consistency of experiment and theory has stringently tested these sectors of the standard model.

Very recently, the Muon $(g-2)$ Collaboration has reported a measurement of the muon's anomalous magnetic moment, which, for the first time, is sensitive to contributions comparable to those of the weak interactions [1]. (See Tables I and II.) The new Brookhaven E821 result is $a_{\mu}^{\exp }=11659202(14)(6) \times 10^{-10}(1.3 \mathrm{ppm})$, where the first uncertainty is statistical and the second is systematic. Combining experimental and theoretical uncertainties in quadrature, the new world average differs from the standard model prediction by $2.6 \sigma$ [1]:

$$
a_{\mu}^{\exp }-a_{\mu}^{\mathrm{SM}}=(43 \pm 16) \times 10^{-10} .
$$

Although of unprecedented precision, the new result is based on a well-tested method used in previous measurements. Polarized positive muons are circulated in a uniform magnetic field. They then decay to positrons, which are emitted preferentially in the direction of the muon's spin. By analyzing the number of energetic positrons detected at positions around the storage ring, the muon's spin precession frequency and anomalous magnetic moment are determined. The new result is based solely on 1999 data. Analysis of the 2000 data is underway, with an expected error of $\sim 7 \times 10^{-10}(0.6 \mathrm{ppm})$, and the final goal is an uncertainty of $4 \times 10^{-10}(0.35 \mathrm{ppm})$ [6].

The standard model prediction has been greatly refined in recent years. The current status is reviewed in Ref. [5] and summarized in Table II. The uncertainty is dominated by hadronic vacuum polarization contributions that enter at 2-loops. This is expected to be reduced by recent measure-
PACS numbers: 13.40.Em, 12.60.Jv, 14.60.Ef, 14.80.Ly

ments of $\sigma\left(e^{+} e^{-} \rightarrow\right.$ hadrons $)$ at center-of-mass energies $\sqrt{s} \sim 1 \mathrm{GeV}$. Thus, although the statistical significance of the present deviation leaves open the possibility of agreement between experiment and the standard model, the prospects for a definitive resolution are bright. If the current deviation remains after close scrutiny and the expected improvements, the anomalous value of $a_{\mu}$ will become unambiguous.

In this paper, we consider the recent measurement of $a_{\mu}$ to be a signal of physics beyond the standard model. In particular, we consider its implications for supersymmetric theories. Supersymmetry is motivated by many independent considerations, ranging from the gauge hierarchy problem, to gauge coupling unification, to the necessity of nonbaryonic dark matter, all of which require supersymmetric particles to have weak scale masses. Deviations in $a_{\mu}$ with the reported magnitude are therefore generic in supersymmetry. In addition, $a_{\mu}$ is both flavor and $C P$ conserving. Thus, while the impact of supersymmetry on other low energy observables can be highly suppressed by scalar degeneracy or small $C P$-violating phases in simple models, supersymmetric contributions to $a_{\mu}$ cannot be. In this sense, $a_{\mu}$ is a uniquely robust probe of supersymmetry, and an anomaly in $a_{\mu}$ is a natural place for the effects of supersymmetry to appear.

The anomalous magnetic moment of the muon is the coefficient of the operator $a_{\mu} \frac{e}{4 m_{\mu}} \bar{\mu} \sigma^{m n} \mu F_{m n}$, where $\sigma^{m n}=\frac{i}{2}\left[\gamma^{m}, \gamma^{n}\right]$. The supersymmetric contribution, $a_{\mu}^{\text {SUSY }}$, is dominated by well-known neutralino-smuon and chargino-sneutrino diagrams [7]. In the absence of significant slepton flavor violation, these diagrams are completely

TABLE I. Recent measurements of $a_{\mu} \times 10^{10}$ and the cumulative world average.

\begin{tabular}{llll}
\hline \hline \multicolumn{1}{c}{ Data set } & \multicolumn{1}{c}{ Result } & World average \\
\hline CERN77 $^{\mathrm{a}}$ & $11659230(85)$ & $(7 \mathrm{ppm})$ & \\
BNL97 $^{\mathrm{b}}$ & $11659250(150)$ & $(13 \mathrm{ppm})$ & $11659235(73)$ \\
BNL98 $^{\mathrm{c}}$ & $11659191(59)$ & $(5 \mathrm{ppm})$ & $11659205(46)$ \\
BNL99 $^{\mathrm{d}}$ & $11659202(14)(6)$ & $(1.3 \mathrm{ppm})$ & $11659203(15)$ \\
\hline \hline
\end{tabular}

${ }^{\text {aRef. [2] }}{ }^{\text {b} R e f . ~[3] ~}{ }^{\text {CRef. [4] }}{ }^{\text {dRef. [1] }}$ 
TABLE II. Contributions to the standard model prediction for $a_{\mu} \times 10^{10}$ (see Ref. [5] and references therein).

\begin{tabular}{lr}
\hline \hline \multicolumn{1}{c}{ Standard model source } & \multicolumn{1}{c}{ Contribution } \\
\hline QED (up to 5-loops) & $11658470.6(0.3)$ \\
Hadronic vac. pol. (2-loop) & $692.4(6.2)$ \\
Hadronic vac. pol. (3-loop) & $-10.0(0.6)$ \\
Hadronic light-by-light & $-8.5(2.5)$ \\
Weak interactions (up to 2-loops) & $15.2(0.4)$ \\
Total & $11659159.7(6.7)$ \\
\hline \hline
\end{tabular}

determined by only seven supersymmetry parameters: $M_{1}, M_{2}, \mu, \tan \beta, m_{\tilde{\mu}_{L}}, m_{\tilde{\mu}_{R}}$, and $A_{\mu}$. The first four enter through the chargino and neutralino masses: $M_{1}, M_{2}$, and $\mu$ are the $\mathrm{U}(1)$ gaugino, $\mathrm{SU}(2)$ gaugino, and Higgsino mass parameters, respectively, and $\tan \beta=\left\langle H_{u}^{0}\right\rangle /\left\langle H_{d}^{0}\right\rangle$ governs gaugino-Higgsino mixing. The last five determine the slepton masses, where $m_{\tilde{\mu}_{L}}$ and $m_{\tilde{\mu}_{R}}$ are the SU(2) doublet and singlet slepton masses, respectively, and the combination $m_{\mu}\left(A_{\mu}-\mu \tan \beta\right)$ mixes left- and right-handed smuons. In general, $M_{1}, M_{2}, \mu$, and $A_{\mu}$ are complex. However, bounds from electric dipole moments typically require their phases to be very small. In addition, $\left|a_{\mu}^{\mathrm{SUSY}}\right|$ is typically maximized for real parameters. In deriving model-independent upper bounds on superparticle masses below, we assume real parameters, but consider all possible sign combinations; these results are therefore valid for arbitrary phases. Our sign conventions are as in Ref. [8].

The qualitative features of the supersymmetric contributions are most transparent in the mass insertion approximation. The structure of the magnetic dipole moment operator requires a left-right transition along the lepton-slepton line. In the interaction basis, this transition may occur through a mass insertion in an external muon line, at a Higgsino vertex, or through a left-right mass insertion in the smuon propagator. The last two contributions are proportional to the muon Yukawa coupling and so may be enhanced by $\tan \beta$. For large and moderate $\tan \beta$, it is not hard to show that the supersymmetric contributions in the mass insertion approximation are all of the form

$$
\frac{g_{i}^{2}}{16 \pi^{2}} m_{\mu}^{2} \mu M_{i} \tan \beta F
$$

where $i=1,2$, and $F$ is a function of superparticle masses, with $F \propto M_{\mathrm{SUSY}}^{-4}$ in the large mass limit [9].

Equation (2) implies $a_{\mu}^{\text {SUSY }} / a_{e}^{\text {SUSY }} \sim m_{\mu}^{2} / m_{e}^{2} \approx 4 \times$ $10^{4} ; a_{\mu}$ is therefore far more sensitive to supersymmetric effects than $a_{e}$, despite the fact that the latter is 350 times better measured. Also, for $M_{2} / M_{1}>0$, although the contributions of Eq. (2) may destructively interfere, typically $\operatorname{sgn}\left(a_{\mu}^{\mathrm{SUSY}}\right)=\operatorname{sgn}\left(\mu M_{1,2}\right)$; we have found exceptions only rarely in highly model-independent scans. Finally, the parameter $\tan \beta$ is expected to be in the range $2.5 \lesssim$ $\tan \beta \lesssim 50$, where the lower limit is from Higgs boson searches, and the upper limit follows from requiring a perturbative bottom quark Yukawa coupling up to $\sim 10^{16} \mathrm{GeV}$. Supersymmetric contributions may therefore be greatly enhanced by large $\tan \beta$.
To determine the possible values of $a_{\mu}^{\text {SUSY }}$ without model-dependent biases, we have calculated $a_{\mu}^{\mathrm{SUSY}}$ in a series of high statistics scans of parameter space. We use exact mass eigenstate expressions for $a_{\mu}^{\text {SUSY }}$. Our calculations agree with Refs. [9-11] and cancel the corresponding standard model diagrams in the supersymmetric limit [12]. We require chargino masses above $103 \mathrm{GeV}$ and smuon masses above $95 \mathrm{GeV}$ [13]. We also assume that the lightest supersymmetric particle (LSP) is stable, as in gravity-mediated theories, and require it to be neutral. Finally, for each scan point we record the mass and identity of the lightest observable supersymmetric particle (LOSP), which we define to be the lightest superpartner with decay products that are detectable at colliders. Given the assumption of a stable LSP, the LOSP is the second lightest supersymmetric particle, or the third if the two lightest are a neutralino and the sneutrino. Note that the identification of supersymmetric events is a complicated and model-dependent issue, especially at hadron colliders. The LOSP, as defined here, is not guaranteed to be observed above background, even if produced.

We begin by scanning over the parameters $M_{2}, \mu, m_{\tilde{\mu}_{L}}$, and $m_{\tilde{\mu}_{R}}$, assuming gaugino mass unification $M_{1}=M_{2} / 2$, $A_{\mu}=0$, and $\tan \beta=50$. The free parameters take values up to $2.5 \mathrm{TeV}$. The resulting values in the ( $\left.M_{\mathrm{LOSP}}, a_{\mu}^{\mathrm{SUSY}}\right)$ plane are given by the points in Fig. 1. We then consider arbitrary (positive and negative) values of $M_{2} / M_{1}$. The resulting values are bounded by the solid curve. As can be seen, and as verified by high statistics sampling targeting the border area, the assumption of gaugino mass unification has no appreciable impact on the envelope curve.

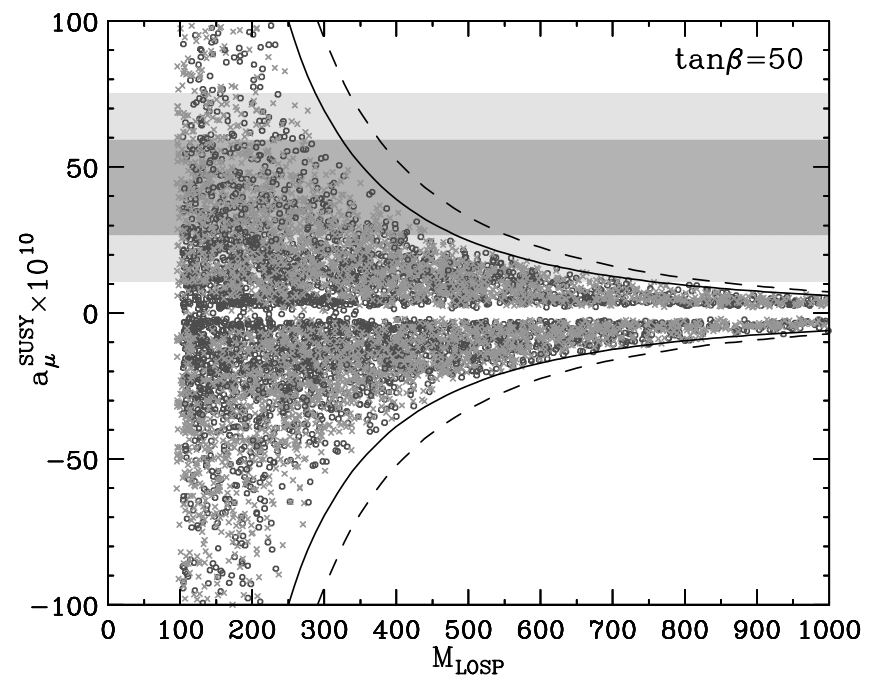

FIG. 1. Allowed values of $M_{\mathrm{LOSP}}$, the mass of the lightest observable supersymmetric particle, and $a_{\mu}^{\mathrm{SUSY}}$ from a scan of parameter space with $M_{1}=M_{2} / 2, A_{\mu}=0$, and $\tan \beta=50$. Crosses (circles) have smuons (charginos/neutralinos) as the LOSP. The $1 \sigma$ - and $2 \sigma$-allowed $a_{\mu}^{\mathrm{SUSY}}$ ranges are indicated. Relaxing the relation $M_{1}=M_{2} / 2$ leads to the solid envelope curve, and further allowing arbitrary $A_{\mu}$ leads to the dashed curve. The envelope contours scale linearly with $\tan \beta$. A stable LSP is assumed. 
Finally, we allow any $A_{\mu}$ in the interval $[-100 \mathrm{TeV}$, $100 \mathrm{TeV}]$. The resulting sample is extremely model independent, and is bounded by the dashed contour of Fig. 1. The envelope contours scale linearly with $\tan \beta$ to excellent approximation.

From Fig. 1 we see that the measured deviation in $a_{\mu}$ is in the range accessible to supersymmetric theories and is easily explained by supersymmetric effects.

The anomaly in $a_{\mu}$ also has strong implications for the superpartner spectrum. Among the most important is that at least two superpartners cannot decouple if supersymmetry is to explain the deviation, and one of these must be charged and so observable at colliders. Nonvanishing $a_{\mu}^{\text {SUSY }}$ thus imply upper bounds on $M_{\text {LOSP. The large value }}$ of $\tan \beta$ is chosen to allow the largest possible $M_{\text {LOSP. The }}$ solid contour is parametrized by

$$
\frac{a_{\mu}^{\mathrm{SUSY}}}{43 \times 10^{-10}}=\frac{\tan \beta}{50}\left(\frac{390 \mathrm{GeV}}{M_{\mathrm{LOSP}}^{\max }}\right)^{2} .
$$

If $a_{\mu}^{\mathrm{SUSY}}$ is required to be within $1 \sigma(2 \sigma)$ of the measured deviation, at least one observable superpartner must be lighter than $490 \mathrm{GeV}(800 \mathrm{GeV})$.

In Fig. 2 we repeat the above analysis, but for the case where the LSP decays visibly in collider detectors, as in models with low-scale supersymmetry breaking or $R$-parity violating interactions. In this case, the LOSP is the LSP. We relax the requirement of a neutral LSP, and require slepton masses above $95 \mathrm{GeV}$ and neutralino masses above $99 \mathrm{GeV}$ [13]. The results are given in Fig. 2. For this case, the solid envelope curve is parametrized by

$$
\frac{a_{\mu}^{\mathrm{SUSY}}}{43 \times 10^{-10}}=\frac{\tan \beta}{50}\left[\left(\frac{300 \mathrm{GeV}}{M_{\mathrm{LOSP}}^{\max }}\right)^{2}+\left(\frac{230 \mathrm{GeV}}{M_{\mathrm{LOSP}}^{\max }}\right)^{4}\right],
$$

and the $1 \sigma(2 \sigma)$ bound is $M_{\mathrm{LOSP}}<410 \mathrm{GeV}(640 \mathrm{GeV})$.

These model-independent upper bounds have many implications. They improve the prospects for the observation of weakly interacting superpartners at the Tevatron

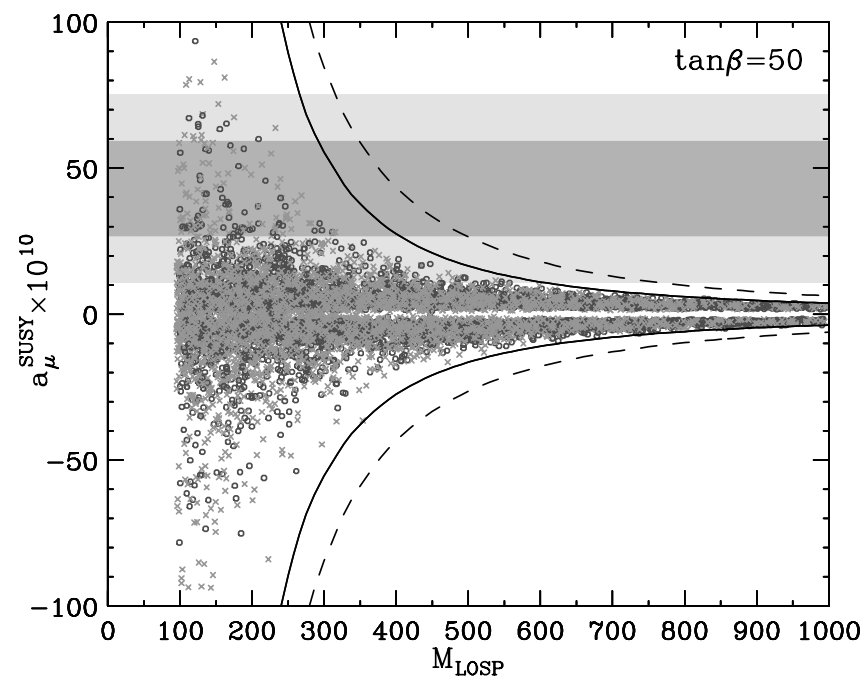

FIG. 2. Same as in Fig. 1, but assuming a visibly decaying LSP. and LHC. They also impact linear colliders, where the study of supersymmetry requires $\sqrt{s}>2 M_{\text {LOSP }}$ (with the possible exception of associated neutralino production in stable LSP scenarios). Finally, these bounds provide fresh impetus for searches for lepton flavor violation, which is also mediated by sleptons and charginos/neutralinos.

We now turn to specific models. The supersymmetric contributions to $a_{\mu}$ have been discussed in various supergravity theories [7], and more recently in models of gaugemediated $[10,14]$ and anomaly-mediated supersymmetry breaking $[8,15]$.

We first consider the framework of minimal supergravity, in which the entire weak scale superparticle spectrum is fixed by four continuous parameters and one binary choice: $m_{0}, M_{1 / 2}, A_{0}, \tan \beta$, and $\operatorname{sgn}(\mu)$, where the first three are the universal scalar, gaugino, and trilinear coupling masses at the grand unified theory (GUT) scale $M_{\mathrm{GUT}} \simeq 2 \times$ $10^{16} \mathrm{GeV}$. We relate these to weak scale parameters through 2-loop renormalization group equations [16] with 1-loop threshold corrections and calculate all superpartner masses to 1-loop [17]. Electroweak symmetry is broken radiatively with a full 1-loop analysis, which determines $|\mu|$.

In minimal supergravity, many potential low energy effects are eliminated by scalar degeneracy. However, $a_{\mu}^{\text {SUSY }}$ is not suppressed in this way and may be large. In this framework, $\operatorname{sgn}\left(a_{\mu}^{\text {SUSY }}\right)=\operatorname{sgn}\left(\mu M_{1,2}\right)$. As is well known, however, the sign of $\mu$ also enters in the supersymmetric contributions to $B \rightarrow X_{s} \gamma$. Current constraints on $B \rightarrow$ $X_{s} \gamma$ require $\mu M_{3}>0$ if $\tan \beta$ is large. In minimal supergravity, then, gaugino mass unification implies that a large discrepancy in $a_{\mu}$ is only possible for $a_{\mu}^{\text {SUSY }}>0$, in accordance with the new measurement.

In Fig. 3 , the $2 \sigma$-allowed region for $a_{\mu}^{\text {SUSY }}$ is plotted for $\mu>0$. Several important constraints are also

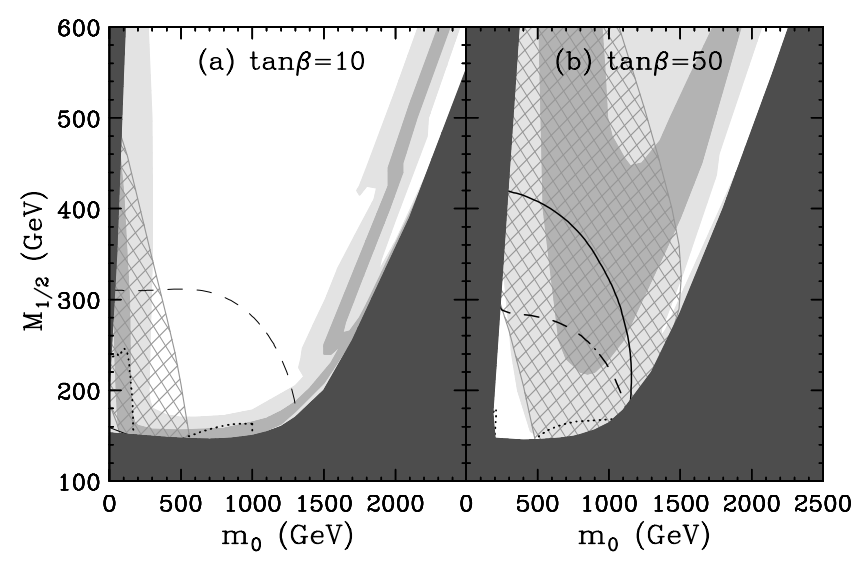

FIG. 3. The $2 \sigma$-allowed region for $a_{\mu}^{\text {SUSY }}$ (hatched) in minimal supergravity, for $A_{0}=0, \mu>0$, and two representative values of $\tan \beta$. The dark regions are excluded by the requirement of a neutral LSP and by the chargino mass limit of $103 \mathrm{GeV}$, and the medium (light) region has LSP relic density $0.1 \leq \Omega h^{2} \leq$ $0.3\left(0.025 \leq \Omega h^{2} \leq 1\right)$. The area below the solid (dashed) contour is excluded by $B \rightarrow X_{s} \gamma$ (the Higgs boson mass), and the regions probed by the trilepton search at Tevatron Run II are below the dotted contours. 
included: bounds on the neutralino relic density, the Higgs boson mass limit $m_{h}>113.5 \mathrm{GeV}$, and the $2 \sigma$ constraint $2.18 \times 10^{-4}<\mathcal{B}\left(B \rightarrow X_{s} \gamma\right)<4.10 \times 10^{-4}$.

For moderate $\tan \beta$, the region preferred by $a_{\mu}^{\mathrm{SUSY}}$ is at low $m_{0}$. Much of the favored region is excluded by the Higgs boson mass. However, the remaining region is consistent with the requirement of supersymmetric dark matter, and, intriguingly, is roughly that obtained in no-scale supergravity [18] and minimal gaugino-mediated [19] models. In contrast, for large $\tan \beta$, there is a large allowed area that extends to large $M_{1 / 2}$ and $m_{0} \approx 1.5 \mathrm{TeV}$, and which also overlaps significantly with a region with desirable relic density. In focus point models with large and universal scalar masses [20], large $\tan \beta$ is therefore favored. The cosmologically preferred regions of minimal supergravity are probed by many pre-LHC experiments [21]. Note, however, that the sign of $\mu$ preferred by $a_{\mu}$ implies destructive interference in the leptonic decays of the second lightest neutralino, and so the Tevatron search for trileptons is ineffective for $200<m_{0}<400 \mathrm{GeV}$ [22].

We close by considering anomaly-mediated supersymmetry breaking [23]. One of the most robust and striking predictions of this framework is that the gaugino masses are proportional to the corresponding beta function coefficients, and so $M_{1,2} M_{3}<0$. Consistency with the $B \rightarrow$ $X_{s} \gamma$ constraint then implies that only negative $a_{\mu}^{\mathrm{SUSY}}$ may have large magnitude, in contrast to the case of conventional supergravity theories $[8,15]$.

In Fig. 4 we investigate how large a positive $a_{\mu}^{\text {SUSY may }}$ be in the minimal anomaly-mediated model. This model is parametrized by $M_{\text {aux }}, m_{0}, \tan \beta$, and $\operatorname{sgn}(\mu)$, where $M_{\text {aux }}$ determines the scale of the anomaly-mediated soft terms, and $m_{0}$ is a universal scalar mass introduced to remove tachyonic sleptons. To get $a_{\mu}^{\mathrm{SUSY}}>0$, we choose $\mu M_{1,2}>0$. We see, however, that the constraint from $B \rightarrow X_{s} \gamma$ is severe, as this sign of $\mu$ implies a construc-

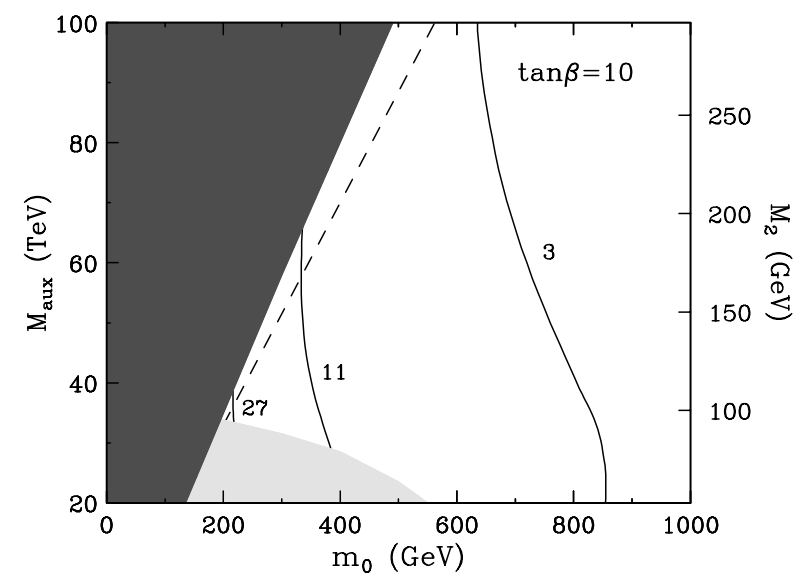

FIG. 4. Contours of $a_{\mu}^{\text {SUSY }} \times 10^{10}$ in the minimal anomalymediated model, for $\mu>0$ and $\tan \beta=10$. The dark region is excluded by $m_{\tilde{\tau}}>82 \mathrm{GeV}$, the light region is excluded at $2 \sigma$ by $\mathcal{B}\left(B \rightarrow X_{s} \gamma\right)<4.10 \times 10^{-4}$, and the LSP is a stau to the left of the dashed line. tive contribution from charginos to $B \rightarrow X_{s} \gamma$ in anomaly mediation. Even allowing a $1 \sigma$ deviation in $a_{\mu}$, we have checked that, for all $\tan \beta$, it is barely possible to obtain $2 \sigma$ consistency with the $B \rightarrow X_{s} \gamma$ constraint. Minimal anomaly mediation is therefore disfavored. The dependence of this argument on the characteristic gaugino mass relations of anomaly mediation suggests that similar conclusions will remain valid beyond the minimal model.

In conclusion, the recently reported deviation in $a_{\mu}$ is easily accommodated in supersymmetric models. Its value provides model-independent upper bounds on masses of observable superpartners and already discriminates between well-motivated models. We await the expected improved measurements with great anticipation.

This work was supported in part by the U.S. Department of Energy under cooperative research agreement DF-FC02-94ER40818.

[1] Muon $(g-2)$ Collaboration, H. N. Brown et al., hepex/0102017.

[2] CERN-Mainz-Daresbury Collaboration, J. Bailey et al., Nucl. Phys. B150, 1 (1979).

[3] R. M. Carey et al., Phys. Rev. Lett. 82, 1632 (1999).

[4] Muon $(g-2)$ Collaboration, H. N. Brown et al., Phys. Rev. D 62, 091101 (2000).

[5] A. Czarnecki and W. J. Marciano, hep-ph/0010194.

[6] R. M. Carey, in Proceedings of the ICHEP00, Osaka, 2000 (to be published); Muon $(g-2)$ Collaboration, R. Prigl et al., hep-ex/0101042.

[7] For references to the early literature, see Ref. [5].

[8] J. L. Feng and T. Moroi, Phys. Rev. D 61, 095004 (2000).

[9] T. Moroi, Phys. Rev. D 53, 6565 (1996); 56, 4424(E) (1997).

[10] M. Carena, G. F. Giudice, and C. E. Wagner, Phys. Lett. B 390, 234 (1997).

[11] T. Blazek, hep-ph/9912460.

[12] S. Ferrara and E. Remiddi, Phys. Lett. 53B, 347 (1974).

[13] See, e.g., LEPFest, CERN, 2000, http://delphiwww.cern.ch/ offline/physics_links/lepc.html

[14] E. Gabrielli and U. Sarid, Phys. Rev. Lett. 79, 4752 (1997); K. T. Mahanthappa and S. Oh, Phys. Rev. D 62, 015012 (2000).

[15] U. Chattopadhyay, D. K. Ghosh, and S. Roy, Phys. Rev. D 62, 115001 (2000).

[16] I. Jack et al., Phys. Rev. D 50, 5481 (1994).

[17] D. M. Pierce, J.A. Bagger, K. Matchev, and R. Zhang, Nucl. Phys. B491, 3 (1997).

[18] A. B. Lahanas and D. V. Nanopoulos, Phys. Rep. 145, 1 (1987).

[19] M. Schmaltz and W. Skiba, Phys. Rev. D 62, 095005 (2000).

[20] J. L. Feng, K. T. Matchev, and T. Moroi, Phys. Rev. Lett. 84, 2322 (2000); Phys. Rev. D 61, 075005 (2000).

[21] J. L. Feng, K. T. Matchev, and F. Wilczek, Phys. Lett. B 482, 388 (2000); Phys. Rev. D 63, 045024 (2001).

[22] K. T. Matchev and D. M. Pierce, Phys. Rev. D 60, 075004 (1999).

[23] L. Randall and R. Sundrum, Nucl. Phys. B557, 79 (1999). 